PROCEEDINGS OF THE

AMERICAN MATHEMATICAL SOCIETY

Volume 132, Number 1, Pages 291-298

S 0002-9939(03)06981-8

Article electronically published on May 7, 2003

\title{
BOUNDING EDGE DEGREES IN TRIANGULATED 3-MANIFOLDS
}

\author{
NOEL BRADY, JON MCCAMMOND, AND JOHN MEIER
}

(Communicated by Ronald A. Fintushel)

\begin{abstract}
In this note we prove that every closed orientable 3-manifold has a triangulation in which each edge has degree 4,5 or 6 .
\end{abstract}

\section{INTRODUCTION}

All closed, orientable 3-manifolds can be constructed in a number of seemingly simple and elegant ways. Examples include Heegaard diagrams, branching over links in the 3-sphere, doing 0/1-Dehn fillings on a cover of the figure 8 knot complement [3], or gluing cubes together so that each edge has degree 3,4 , or 5 (see [1]). Recall that the degree of an edge $e$ in a 3-complex is the number of closed 3-cells which contain $e$. In this article we add a new construction to this list: gluing tetrahedra together so that each edge has degree 4,5 , or 6 . Our primary motivation for this research comes from recent work on triangulated 3-manifolds with restricted edge degrees. See [2] and [5].

As in the construction of Cooper and Thurston [1], our construction relies heavily on the universality of the figure 8 knot complement. Specifically, we give explicit triangulations of a solid torus $\mathbb{S}^{1} \times D^{2}$, a thickened torus $T^{2} \times[0,1]$, and the complement of the figure 8 knot. Each of these triangulations will be acceptable in the following sense.

Definition 1.1 (Acceptable triangulations). Let $M$ be an triangulated, orientable 3 -manifold, possibly with boundary. If each interior edge has degree 4, 5, or 6 and every boundary edge has degree 2 or 3 , then we will say that the triangulation of $M$ is acceptable.

Notice that identifying two manifolds with acceptable triangulations along boundary components will produce a new manifold with an acceptable triangulation. Gluing covers of these three constructions together will prove our main result:

Theorem 1.2. Every closed orientable 3-manifold $M$ has an acceptable triangulation. In particular, there exists a triangulation of $M$ in which every edge has degree 4,5 or 6 .

Received by the editors January 14, 2002 and, in revised form, August 8, 2002.

2000 Mathematics Subject Classification. Primary 57Q15, 57M12.

Key words and phrases. 3-manifold, triangulation, branched covering.

The first author was partially supported under NSF grant no. DMS-9996342.

The second author was partially supported under NSF grant no. DMS-9971682. 


\section{SOLID TORI}

In this section we describe an explicit triangulation of the solid torus $\mathbb{S}^{1} \times D^{2}$, but first we need to establish some notation for marked triangulated tori.

Definition 2.1 (Standard triangulation). Let $\mathbb{Z}[\omega]=\{a+b \omega \mid a, b \in \mathbb{Z}\} \subset \mathbb{C}$ be the lattice in the complex plane where $\omega$ is the cube-root of unity, $\frac{1}{2}+\frac{\sqrt{3}}{2} i$. The standard triangulation of $\mathbb{C}$ will be the triangulation where the vertices are the elements of $\mathbb{Z}[\omega]$ and the edges connect vertices when they are distance 1 apart. See Figure [1]

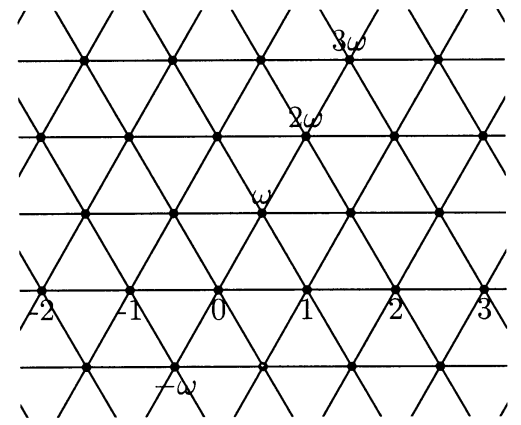

FiguRE 1. $\mathbb{Z}[\omega]$

Definition 2.2 ((u,v)-torus). Recall that a marked torus is a torus $T$ together with an ordered pair of elements $(a, b)$ which generate $\pi_{1}(T)$, and let $T$ be a marked, triangulated torus whose universal cover is explicitly identified with the standard triangulation of $\mathbb{C}$ by a map $f: \widetilde{T} \rightarrow \mathbb{C}$. The elements $a$ and $b$ will act on this standard triangulation by deck transformations and, in particular, they will correspond to maps $z \mapsto z+u$ and $z \mapsto z+v$ where $u$ and $v$ are elements of $\mathbb{Z}[\omega]$. A marked triangulated torus with an explicit identification of this type will be called a $(u, v)$-torus.

Definition 2.3 (Triangular columns). A triangular column is constructed as follows. The vertices are labeled by the integers, and every four consecutive integers form the vertices of a tetrahedron. These are the only tetrahedra in the complex. A concrete realization of this complex in $\mathbb{C} \times \mathbb{R}$ can be obtained by assigning the label $3 k$ to $(0,3 k), 3 k+1$ to $(1,3 k+1)$, and $3 k+2$ to $(\omega, 3 k+2)$. A triangular column is shown in Figure 2 where the real direction is horizontal. Note that the edges in a triangular column have degree 1 if its vertex labels differ by exactly 3 , and degree 2 otherwise. Moreover, the degree 2 edges form three spirals in this realization. There is one spiral whose consecutive vertex labels differ by 1 , and there are two parallel spirals whose consecutive vertex labels differ by 2 . The reader should note that this column in $\mathbb{C} \times \mathbb{R}$ has slightly different combinatorial properties than its mirror image. In particular, the spiral whose consecutive vertex labels differ by 1 will spiral in the other direction in its mirror image. We will call the two types of columns left-handed columns and right-handed columns depending on the direction of the spiral whose consecutive vertex labels differ by 1 . The distinction will be important when we start to glue columns together. 


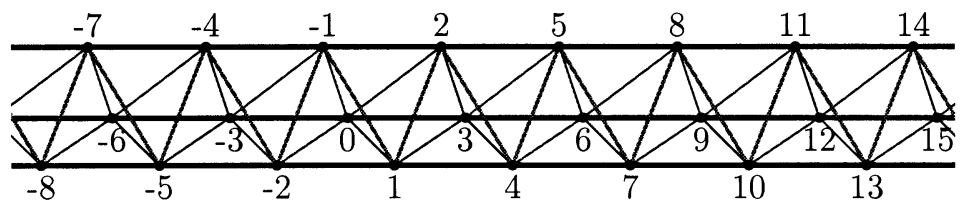

Figure 2. A right-handed triangular column.

Definition 2.4 (Triangular rings). If $\ell$ is a positive integer, then there is a translation of a triangular column which sends vertex $k$ to $k+3 * \ell$. Quotienting by this translation gives a solid torus which we will call a triangular ring of length $\ell$. As with columns, there are left-handed and right-handed rings. It is easy to see that the boundary of a ring marked by its meridian and longitude is a $(3 \omega-1, \ell)$-torus, that all edges are boundary edges, and that all edges have degree 2 except for three circles of edges with degree 1 which run along the longitude. These three circles will be called the edges of the ring. Moreover, the edges of the ring cut the boundary torus into three annuli with identical combinatorial patterns. We will call these the faces of the ring. Since there is a unique combinatorial pattern on the face of a ring of length $\ell$, any two faces on distinct rings of length $\ell$ can be identified in an essentially unique way.

Definition 2.5 (Strips). A strip $S$ of length $\ell$ is the solid torus which results when a face of a triangular ring of length $\ell$ is attached to a face of its mirror image. The boundary of the strip marked by its meridian and longitude is a $(2 \sqrt{-3}, \ell)$-torus, every interior edge has degree 4 , and all boundary edges are degree 2 except for two circles of edges with degree 1 which run along the longitude. As in the case of rings we will call these two circles the edges of the strip. The edges of the strip cut the boundary torus into two annuli with common boundaries - called the sides of the strip - and there is an obvious identification between these sides which fixes the edges and preserves the combinatorial structures of the sides. Notice that this would not be the case if we glued two left-handed rings together or two right-handed rings. Because of this property, attaching a strip to the boundary of a 3-manifold along its side will not change the combinatorial pattern of the boundary.

Lemma 2.6 (Solid tori). If $k$ and $\ell$ are arbitrary positive integers, there is an acceptable triangulation of $\mathbb{S}^{1} \times D^{2}$ where the boundary torus marked by its meridian and longitude is a $(3 k \sqrt{-3}, \ell)$-torus.

Proof. Glue a number of triangular rings of length $\ell$ together along their faces so that its cross section looks like the portion of the standard triangulation of $\mathbb{C}$ bounded by $0,2 k, k+k \omega$ and $k \omega$. The cross section for $k=4$ is shown in Figure 3 Then glue this construction to its mirror image so that the faces corresponding to the line from 0 to $2 k$ in the cross section are the faces that are glued to their mirror images. Because we are using the mirror image of the construction, when the longitude is identified with the real number $\ell$, the meridian will be purely imaginary. In particular, the boundary torus marked by its meridian and longitude will be a $(3 k \sqrt{-3}, \ell)$-torus. 


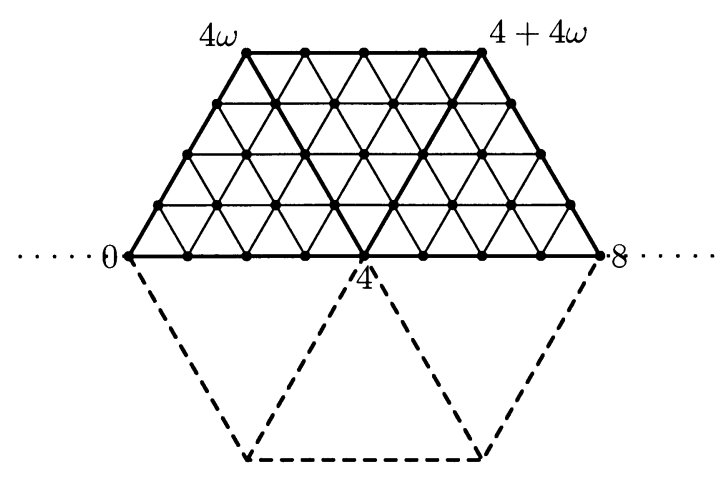

Figure 3. Cross-section of half the solid torus constructed in Lemma 2.6] when $k=4$.

\section{THICKENED TORI}

In this section we describe a triangulation of the thickened torus $T^{2} \times[0,1]$. Before stating its properties, we should note that if $T^{2}$ is a marked torus, then $T^{2} \times\{0\}$ and $T^{2} \times\{1\}$ inherit markings from the obvious identification of their fundmental groups.

Lemma 3.1 (Thickened-torus). If $T^{2}$ is a marked torus and $k$ and $\ell$ are positive integers, then there is an acceptable triangulation of $T^{2} \times[0,1]$ where $T^{2} \times\{0\}$ is a $(k \sqrt{-3}, \ell)$-torus and $T^{2} \times\{1\}$ is a $(k, \ell \sqrt{-3})$-torus.

Proof. We start by explicitly triangulating $\mathbb{C} \times[0,1]$ as follows. Let $\mathbb{Z}[i]=\{a+$ $b i \mid a, b \in \mathbb{Z}\}$ be the Gaussian integers. The vertices of the triangulation will be $V_{0} \cup V_{1}$ where $V_{0}=\{(u, 0) \mid u \in \mathbb{Z}[i]\}$ and $V_{1}=\left\{(u, 0)+\left(\frac{1}{2}+\frac{1}{2} i, 1\right) \mid u \in \mathbb{Z}[i]\right\}$. There are three types of edges. The first type connect $v$ to $v+(1,0)$ and $v$ to $v+(i, 0)$ for each vertex $v$. These edges slice $\mathbb{C} \times\{0\}$ and $\mathbb{C} \times\{1\}$ into squares. The second type connect $v$ to $v+\left( \pm \frac{1}{2}+ \pm \frac{1}{2} i, 1\right)$ for each choice of sign and for each $v \in V_{0}$. The slab $\mathbb{C} \times[0,1]$ can now be viewed as a union of square-based pyramids where each base lies in a boundary plane. The third type of edge splits these pyramids into pairs of tetrahedra. For each $v \in V_{0}$ connect $v$ to $v+(1-i, 0)$ and for each $v \in V_{1}$ connect $v$ to $v+(1+i, 0)$. A top view of this construction is shown in Figure 4, It is easy to check that this triangulation is acceptable. To complete the construction we quotient $\mathbb{C} \times[0,1]$ by the translations $(z, t) \mapsto(z+k+k i, t)$ and $(z, t) \mapsto(z+\ell-\ell i, t)$, using these two deck transformations to mark the resulting thickened torus.

Corollary 3.2. If $k$ and $\ell$ are arbitrary positive integers, there is an acceptable triangulation of $\mathbb{S}^{1} \times D^{2}$ where the boundary torus marked by its meridian and longitude is a $(3 k, \ell \sqrt{-3})$-torus.

Proof. By Lemma 2.6. there is a solid torus $M$ marked by its meridian and longitude whose boundary is a $(3 k \sqrt{-3}, \ell)$-torus. Also, by Lemma 3.1, there is a thickened marked torus whose boundary components are a $(3 k, \ell \sqrt{-3})$-torus and a $(3 k \sqrt{-3}, \ell)$-torus, respectively. Gluing the solid marked torus to the thickened marked torus along their $(3 k \sqrt{-3}, \ell)$-torus boundary components completes the construction. The resulting triangulation remains acceptable. 


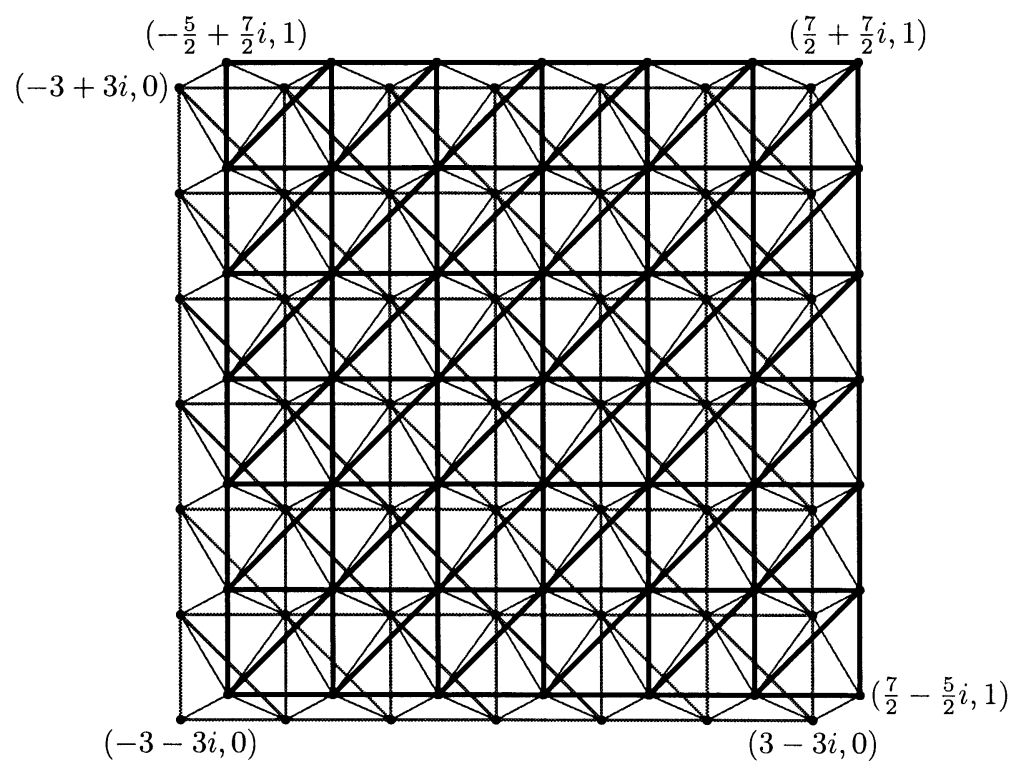

Figure 4. A triangulation of $\mathbb{C} \times[0,1]$. The thick "black" lines lie in $\mathbb{C} \times\{1\}$, the medium "red" lines in $\mathbb{C} \times\{0\}$, and the thin "blue" lines are interior.

\section{Figure 8 KNOT}

Let $M_{8}$ denote the 3-manifold with boundary which is obtained by removing an open tubular neighborhood of the figure 8 knot in the 3 -sphere. It is well known that $M_{8}$ is homeomorphic to a cell complex consisting of two truncated tetrahedra which are glued along their hexagonal faces and that the boundary torus of this complex, as marked by the meridian and longitude of the removed solid torus, is a $(1,2 \sqrt{-3})$-torus. See Figure 5 for the gluing diagram and see [4] or [6] for further details. For later reference we note here that the 1-skeleton of a $(1,2 \sqrt{-3})$-torus consists of 6 geodesic circles. There are 4 circles of length 1 parallel to the meridian and there is a single circle of length 4 in each of the other two remaining directions.
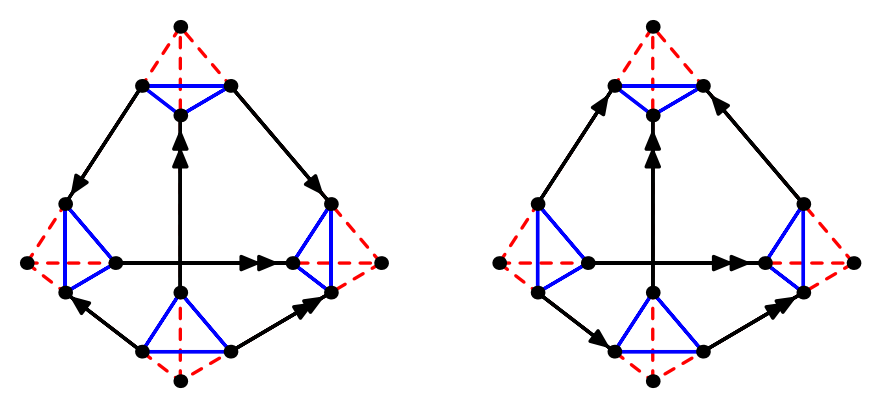

Figure 5. The gluing diagram for $M_{8}$. 
In this section we create an acceptable triangulation for $M_{8}$ in two stages: we start by triangulating its "octahedral core" and then we will attach several "curvature averaging strips". The first step is easiest to describe by explicitly describing how to truncate tetrahedra.

Definition 4.1 (Truncating tetrahedra). Let $e_{i}, i=1, \ldots, 4$, be the standard basis vectors in $\mathbb{R}^{4}$. One description of a 3 -simplex $\Delta$ is all the points in $\mathbb{R}^{4}$ of the form $\sum_{i=1}^{4} t_{i} e_{i}$ with $0 \leq t_{i} \leq 1$. To form an ideal tetrahedron we simply require $t_{i}<1$ for all $i$. Further restricting the $t_{i}$-values produces truncated tetrahedra and eventually a degenerate octahedron. More precisely let $\Delta_{s}$ be the portion of the 3 -simplex in which each $t_{i}$ is at most $s, s \in[1 / 2,1)$. For each $s \in\left(\frac{1}{2}, 1\right), \Delta_{s}$ is a truncated tetrahedron, and for $s=\frac{1}{2}, \Delta_{s}$ is an octahedron. See Figure 6 .
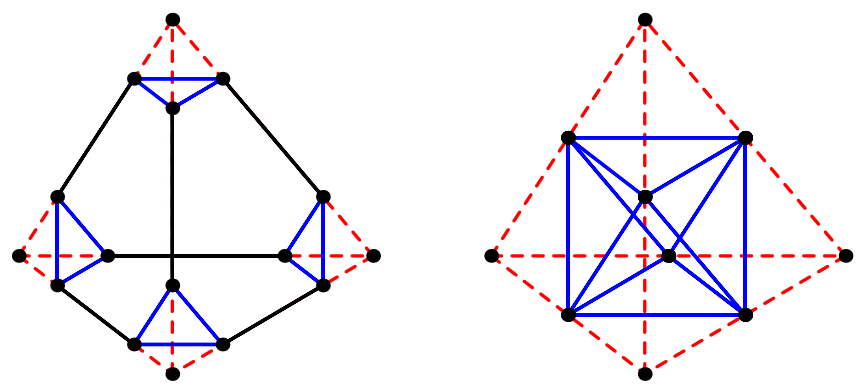

Figure $6 . \Delta_{s}$ for $s=\frac{3}{4}$ and $s=\frac{1}{2}$.

Definition 4.2 (Octahedral core). Note that we can create a deformation retraction which shrinks $\Delta_{s}$ to $\Delta_{s^{\prime}}$ and preserves the face structures for all $s>s^{\prime} \geq \frac{1}{2}$. Pasting these retractions together, we can define a retraction from $M_{8}$ to a complex built out of two octahedra. We call the result the octahedral core of $M_{8}$.

Notice that the octahedral core is not quite a 3-manifold since each of the two points corresponding to baricenters of the edges of the original ideal tetrahedra has a neighborhood which is homeomorphic to a cone on an annulus. Similarly, the boundary of the core is not quite a torus. As $s$ decreases to $\frac{1}{2}$ and $M_{8}$ retracts to its core, two pairs of points on its boundary torus are identified. We think of this singular torus as the "boundary" of the octahedral core. The only curves in the boundary of the core we will consider are those which are retractions of curves in the boundary of $M_{8}$, and such a curve will be described as simple if and only if it is the retraction of a simple curve in the boundary of $M_{8}$. We are now ready to triangulate $M_{8}$.

Lemma 4.3 (Figure 8 knot complement). For each integer $k \geq 3$, the figure 8 knot complement $M_{8}$ has an acceptable triangulation whose boundary torus marked by the standard meridian and longitude is a $(k, 2 k \sqrt{-3})$-torus.

Proof. We start with the octahedral core and we triangulate each face of each octahedra by subdividing it so that it looks like the standard triangulation of $\mathbb{C}$ in the triangle bounded by $0, k$ and $k \omega$. In particular, every edge of the core is subdivided into $k$ edges and every 2 -cell in the core is subdivided into $k^{2}$ small 
triangles. Next, we triangulate the interior of each octahedron by coning off its triangulated boundary to its barycenter. Notice that the "boundary torus" has become a $(k, 2 k \sqrt{-3})$-torus as a result of the subdivision.

Doing this subdivision to a single octahedron creates internal edges with degrees 4 and 6 and boundary edges of degree 2 . Thus in the core internal edges have degree 4 or 6 , and boundary edges have degree 2 or 4 . Moreover, the boundary edges of degree 4 arise from the edges of the original octahedra prior to the subdivision. Thus they line up to form 6 circles, corresponding to the 6 circles in a $(1,2 \sqrt{-3})$ torus. These 6 circles form one group of 4 parallel circles, all of length $k$, and two circles of length $4 k$.

To make the triangulation acceptable we now attach a "curvature averaging strip" to an annular neighborhood of each of these 6 circles. We will add the strips in one direction at a time, so that the result looks like a traditional apple pie crust. The process is illustrated in Figure 7 . Combinatorially, the effect of attaching such a strip is to replace an annular neighborhood of the circle by a combinatorially identical annulus. The degree of the central circle drops from 4 to 2 , while the degree of the neighboring circles increases by 1 . Since we have assumed that $k \geq 3$, the edges in these neighboring circles have degree 2 and they are distinct from each other. The degrees of the edges which have been covered by the strip are 4,5 or 6 since they initially had degrees between 2 and 4 and the edges in the strip which were attached to them all had degree 2 .

When we attach the first strip, some of the other circles of edges of degree 4 will become broken circles, but the key observation is that all of the degrees of boundary edges remain 2, 3, or 4, and the circles on either side of a (broken) circle of edges of degree 4 remain of degree 2 . Thus when a strip is added to cover up these edges of degree 4 , no new edges of degree 4 will be created. Finally, note that we chose $k \geq 3$ so that parallel strips would not have boundary circles in common.

The net effect of adding all of these strips is to thicken the octahedral core into a 3-manifold with boundary homeomorphic to $M_{8}$ and to make the resulting triangulation acceptable. In addition, the boundary torus has remained a $(k, 2 k \sqrt{-3})$-torus throughout the process.

\section{Construction}

Combining the previous constructions immediately yields our main result.

Theorem 1.2, Every closed orientable 3-manifold $M$ has an acceptable triangulation. In particular, there exists a triangulation of $M$ in which every edge has degree 4,5 or 6 .

Proof. By the universality of the figure 8 knot [3], $M$ can be obtained by doing $0 / 1$ Dehn fillings of each cusp of a finite cover $M^{\prime}$ of the figure 8 knot complement. By lifting the triangulation described in Lemma 4.3 (for $k=3$ ) through this finite cover, we get an acceptable triangulation of $M^{\prime}$. Let $T^{2}$ be one of the torus cusps of $M^{\prime}$ marked by its meridian and longitude. Because this is a covering map, there are positive integers $k$ and $\ell$ such that $T^{2}$ is a $(3 k, 6 \ell \sqrt{-3})$-torus. By Corollary 3.2 there is an acceptable triangulation of a solid torus marked by its meridian and longitude whose boundary is also a $(3 k, 6 \ell \sqrt{-3})$-torus. Attaching these two marked tori along their common combinatorial pattern completes the $0 / 1$ filling. The resulting triangulation remains acceptable and filling each of the cusps in this fashion completes the construction. 

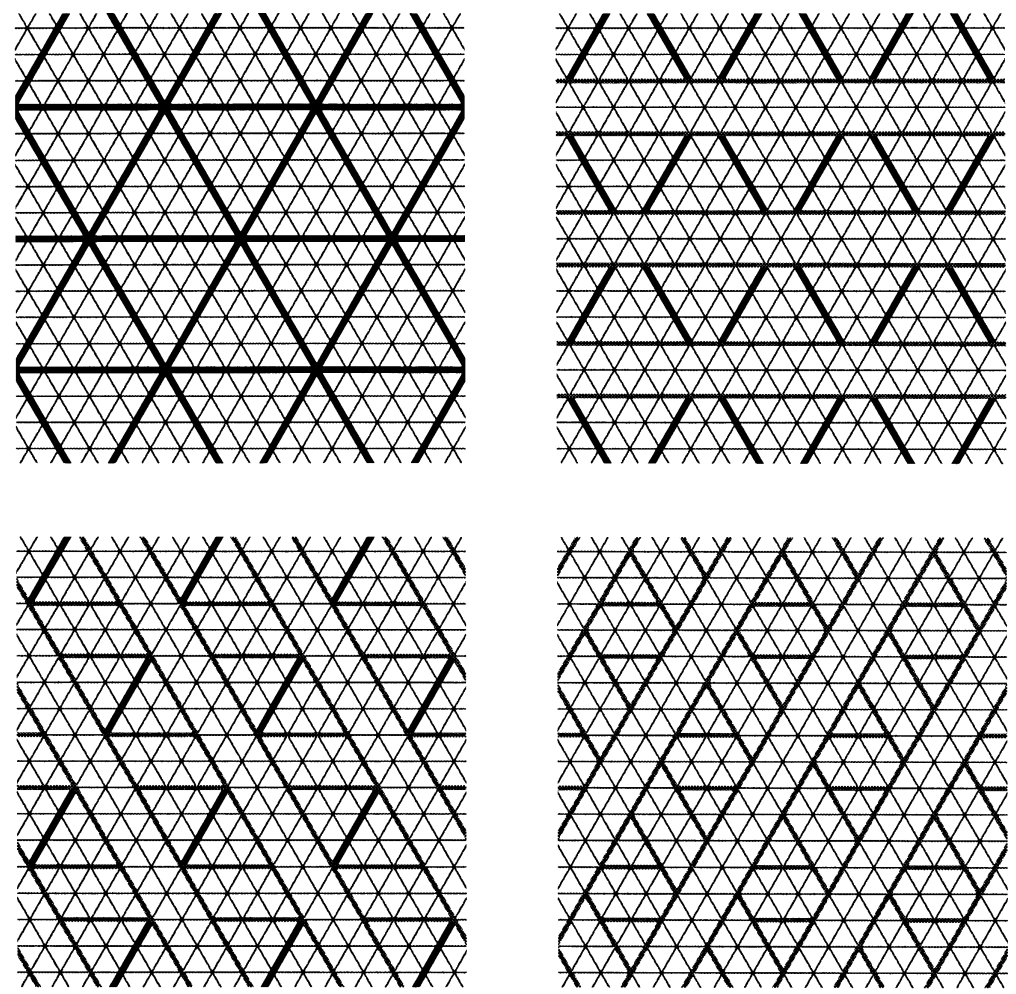

FiguRE 7 . The boundary torus as the various strips are added. The thick "black" lines represent edges with degree 4, the medium "red" lines represent edges of degree 3 , and the thin "blue" lines represent edges of degree 2 . In this example $k=5$.

\section{REFERENCES}

1. Daryl Cooper and William P. Thurston, Triangulating 3-manifolds using 5 vertex link types, Topology 27 (1988), no. 1, 23-25. MR 89d:57028

2. Murray Elder, Jon McCammond, and John Meier, 3-manifold triangulations and wordhyperbolicity, In preparation.

3. Hugh M. Hilden, María Teresa Lozano, and José María Montesinos, On knots that are universal, Topology 24 (1985), no. 4, 499-504. MR 87a:57010

4. John G. Ratcliffe, Foundations of hyperbolic manifolds, Springer-Verlag, New York, 1994. MR 95j:57011

5. John Sullivan, New tetrahedrally close-packed structures, Preprint 2000.

6. William P. Thurston, The geometry and topology of three-manifolds, Princeton lecture notes, 1978-80.

Department of Mathematics, University of Oklahoma, Norman, Oklahoma 73019

E-mail address: nbrady@math.ou.edu 93106

Department of Mathematics, University of California, Santa Barbara, California

E-mail address: jon.mccammond@math.ucsb.edu

Department of Mathematics, Lafayette College, Easton, Pennsylvania 18042

E-mail address: meierj@lafayette.edu 